DESY preprint 05-069

\title{
Vortices on the worldsheet of the QCD string
}

\author{
Harvey B. Meyer \\ DESY-Zeuthen, \\ Platanen Allee 6 \\ D-15738 Zeuthen \\ harvey.meyer@desy.de
}

\begin{abstract}
We investigate the properties of the QCD string in the Euclidean $\mathrm{SU}(N)$ pure gauge theory when the space-time dimensions transverse to it are periodic. From the point of view of an effective string theory, the string tension $\sigma$ and the low-energy constants $c_{k}$ of the theory are arbitrary functions of the sizes of the transverse dimensions $L_{\perp}$. Since the gauge theory is linearly confining in $D=2,3$ and 4 dimensions, we propose an effective string action for the flux-tube energy levels at any choice of $L_{\perp}$, given $\sigma\left(L_{\perp}\right)$ and $c_{k}\left(L_{\perp}\right)$. The Lüscher term only depends on the number of massless bosonic degrees of freedom and the effective theory can account for its evolution as a function of $L_{\perp}$. As the size of one transverse dimension is varied, we predict a Kosterlitz-Thouless transition of the worldsheet field theory at $\sigma\left(L_{\perp}\right) L_{\perp}^{2} \simeq 1 / 8 \pi$ driven by vortices, after which the periodic component of the worldsheet displacement vector develops a mass gap and the effective central charge drops by one unit. The universal properties of the transition are emphasised.
\end{abstract}




\section{Introduction}

Consider the Euclidean $\mathrm{SU}(N)$ pure gauge theory on a four-dimensional hypertorus of size $\left(L_{1}, L_{2}, L_{3}, L_{4}\right)$, with a pair of Polyakov loops running along the direction 4. $L_{3}$ and $L_{4} \equiv L$ are fixed but very large compared to all other length scales in the setup. Direction 4 is interpreted as the time direction, so that the potential between two static sources can be extracted from the Polyakov loop correlator:

$$
\left\langle P_{4}(0) P_{4}^{*}\left(R \hat{e}_{3}\right)\right\rangle \equiv e^{-V\left(R ; L_{1}, L_{2}\right) L}, \quad L \gg R .
$$

We are now interested in the system as a function of the size of the two other dimensions, $L_{1}$ and $L_{2}$. If one of them is kept large while the other is shrunk to zero, we will observe the 'deconfining phase transition', where the centre symmetry along the finite dimension breaks spontaneously; decreasing it further, eventually the theory dimensionally reduces to a three-dimensional $\mathrm{SU}(N)$ gauge theory. If we now follow the same evolution, gradually reducing another dimension, we again go through a deconfining phase transition, followed by a dimensional reduction to a two-dimensional theory. At this point, the pair of Polyakov considered above loops still obeys an area law; the theory is now perturbatively solvable.

In fact, at any stage of this two-step dimensional reduction, the Polyakov loop correlator obeys an area law characterised by a string tension $\sigma\left(L_{1}, L_{2}\right)$ varying with the size of the transverse dimensions:

$$
V\left(R ; L_{1}, L_{2}\right) \sim \sigma\left(L_{1}, L_{2}\right) R, \quad \sigma\left(L_{1}, L_{2}\right) R^{2} \gg 1
$$

An effective low-energy theory was recently proposed by Lüscher and Weisz [6] to describe the corrections to the classical string behaviour $V(R)=\sigma R$, for the case of infinite transverse dimensions in the three- and four-dimensional $\mathrm{SU}(N)$ gauge theories. The central idea is to equate the Polyakov loop correlator with the partition function of the fluctuating $L \times R$ surface, corresponding to the string worldsheet. The leading correction to the ground state energy ${ }^{1}$ comes from Gaussian fluctuations:

$$
V(R)=\sigma R-\frac{\pi d_{\perp}}{24 R}+O\left(\frac{1}{\sigma R^{3}}\right)
$$

where $d_{\perp}$ is the number of (infinite) transverse space-time dimensions. It is the famous Lüscher correction 4, in favour of which a significant amount of numerical evidence has been gathered [3, 10].

Since we have a linearly rising potential at any choice of $\left(L_{1}, L_{2}\right)$, we can attempt to write an effective string theory for every $\left(L_{1}, L_{2}\right)$. As the latter are varied, we expect the effective theory to interpolate between the purely four-dimensional, the threedimensional and the (trivial) two-dimensional case. The unknown parameters of the effective string theory now become functions of $\left(L_{1}, L_{2}\right)$.

When the transverse dimensions are large, $\sigma$ becomes independent of their size. As $L_{1}$ shrinks to zero, $\sigma \propto 1 / L_{1}^{2}$ (by asymptotic freedom); when $L_{2} \rightarrow 0$ as well, $\sigma$ is proportional to $1 /\left(L_{1} L_{2}\right)$. We note that to discuss a smooth evolution of $V(R)$ as a function of $\left(L_{1}, L_{2}\right)$, we must measure $R$ and $V(R)$ at each $\left(L_{1}, L_{2}\right)$ in units of

\footnotetext{
${ }^{1}$ In this paper we shall be interested only in the ground state of the quark-antiquark system, whose contribution dominates the correlator at large enough $L$.
} 
$\sigma\left(L_{1}, L_{2}\right)$. From the point of view of the four-dimensional theory, the increase of the latter as the transverse volume decreases can be interpreted in terms of the overlap around the transverse dimensions of the chromoelectric flux lines. Assuming that the flux-tube energy density falls off transversely as $e^{-m_{G} r_{\perp}}$, where $m_{G}$ is the scalar glueball mass (something that is hard to establish numerically [19]), the corrections on $\sigma\left(L_{1}, L_{2}\right)$ would be $O\left(e^{-m_{G} L_{\min }}\right)$, when $L_{\min }=\min \left(L_{1}, L_{2}\right)$ is large.

In addition, there are the 'low-energy constants' $c_{k}$ appearing in the effective action describing the energetics of the worldsheet fluctuations beyond the quadratic order. These functions of $\left(L_{1}, L_{2}\right)$ have to be given as input in the effective theory. The only limit in which they are known is the $L_{1}, L_{2} \rightarrow 0$ limit, where they vanish.

However, the Lüscher correction is completely independent of both $\sigma$ and the $c_{k}$. The question that motivates the present paper is then the following. When the transverse dimensions $L_{1}, L_{2}$ are large, the coefficient of the Lüscher correction for a bosonic string is $-\pi / 12$; when a transverse dimension shrinks to zero, the gauge theory should dimensionally reduce to a three-dimensional one, so the Lüscher coefficient is then only $-\pi / 24$. The $1 / R$ correction disappears altogether when a second dimension is shrunk to zero. How and at what temperature do these transitions occur? From the point of view of the effective string theory, the coefficient is nothing but the central charge of the $2 \mathrm{~d}$-conformal theory describing the fluctuations of the worldsheet; it effectively counts the number of massless bosonic degrees of freedom living on the worldsheet. We thus expect the Lüscher correction to have a discontinuity at a phase transition of the worldsheet quantum field theory.

The component along a compact direction (say that of size $L_{1}$, while $L_{2}$ stays large) of the worldsheet field $h\left(z_{0}, z_{1}\right)$ that describes its fluctuations becomes a periodic variable. Since the worldsheet field theory is treated as an effective theory with a fixed cutoff, it can be described as living on a lattice with a fixed lattice spacing of order $\sigma^{-1 / 2}$. One is then led to consider, as effective partition function, the class of statistical mechanics models which share the main features of the famous XY-model [8]. In particular the vibrational modes of the string are expected to undergo a phase transition of the Kosterlitz-Thouless (KT) type [8] related to the proliferation of vortices on the string worldsheet. They have the effect of disordering the fluctuations by generating a finite screening length. These vortices are interpreted as tunnelling amplitudes between classical configurations of the string with different winding numbers. The KT criterion, based on an energy vs. entropy argument, yields an estimate of the gauge-theory temperature where the transition occurs (see section 4),

$$
T^{*} \equiv 1 / L_{1}^{*} \simeq \sqrt{\frac{\sigma}{8 \pi}} .
$$

The precise value of this temperature is non-universal; in particular, the string tension appearing in the formula is $\sigma\left(L_{1}, L_{2}\right)$ and differs by a factor of order one from the string tension at $L_{1}=\infty$. Since the high-temperature phase of the XY model is dominated by vortex configurations and admits a finite mass gap (even in the infinite worldsheetvolume limit), this transition naturally explains the disappearance of the universal string correction associated with the periodic component of the worldsheet field $h$. One of the aims of this paper is to describe the signatures of such a Kosterlitz-Thouless transition in the spectrum of the QCD flux-tube. In this respect, one might worry that $T^{*}$ is parametrically of order one in units of the string tension, so that, in the four-dimensional 
language, there is no parametric suppression of the string internal degrees of freedom at that temperature. But these effects are described in our framework through the dependence of $\sigma$ and the $c_{k}$ on the size of the transverse dimension.

The rest of this paper is organised as follows. In section 2 we review the properties of the 2D XY model relevant for our discussion of the effective worldsheet theory. The notation for the latter are set in section 3 , and section 4 describes the main results on the effect of a finite periodic transverse dimension. A summary and outlook follows.

\section{On the Kosterlitz-Thouless phase transition}

We review some well-known facts about the XY-model and the Kosterlitz-Thouless phase transition. For more details, we refer the reader to the abundant literature on the subject [12, 13, 14, 15.

We take as starting point the $O(2)$ non-linear $\sigma$-model. Once the 2-component field is parametrised as $(\cos \theta, \sin \theta)$, the continuum action is deceptively simple:

$$
S=\frac{\kappa}{2} \int d^{2} x \partial_{\mu} \theta \partial_{\mu} \theta
$$

What makes the dynamics non-trivial is that $\theta$ is an angle variable. Therefore it cannot be rescaled, and $\kappa$ is not a redundant variable. The (IR-regularised) two-point function in the Gaussian approximation is thus given by $-\frac{1}{2 \pi \kappa} \log \left(\frac{r}{r_{o}}\right)$. Such a perturbative analysis around the trivial vacuum $\theta \equiv 0$ of course does not take the periodicity into account, and therefore the dynamics is that of an ordinary massless scalar field in that approximation. A convenient way to deal with the periodicity of $\theta$ is to let it take values in $\mathbf{R}$ but identify points differing by $2 \pi$. This means that a configuration of $\theta$ can be multivalued, as long as $e^{i \theta}$ is not.

The classical equation of motion $\Delta \theta=0$, together with the boundary condition that $\theta$ goes to a constant at infinity, implies that either $\theta$ is constant or has singularities. If the latter are isolated, the most general solution is 13 .

$$
e^{i \theta(z)}=e^{i \theta_{\infty}} \prod_{i=1}^{n} \frac{\left(z-z_{i}^{+}\right) /\left|z-z_{i}^{+}\right|}{\left(z-z_{i}^{-}\right) /\left|z-z_{i}^{-}\right|}
$$

The interpretation of the solution is that vortices of intensity \pm 1 are centred at the points $z_{i}^{ \pm}$. Higher intensities are obtained when several $z_{i}^{+}$(or several $z_{i}^{-}$) coincide. The angle $\theta$ is a multivalued function, but $e^{i \theta}$ is well-defined everywhere, except at the singular points.

Although the energy of vortex configurations is divergent both in the infrared and in the ultraviolet, in the (lattice) regularised theory their entropy is of the same functional form $^{2} \log L / a$. So, depending on the temperature, they can play an important role in the partition function. The estimate for the critical temperature one obtains from this Kosterlitz-Thouless criterion is

$$
\kappa^{*}=\frac{2}{\pi}
$$

\footnotetext{
${ }^{2} L$ is the linear size of the system; the energy of a vortex-antivortex pair is roughly obtained by replacing $L$ by their separation.
} 
These considerations show that to compute the corrections to the free theory, we need to formulate it on a lattice and generalise the perturbative approach by taking into account Gaussian fluctuations around not only the trivial vacuum, but also the vortex configurations:

$$
S\left[\theta=\theta_{\text {vort }}+\delta \theta\right]=S_{\text {vort }}\left[\theta_{\text {vort }}\right]+\frac{\kappa}{2} \int d^{2} x\left(\partial_{\mu}(\delta \theta)\right)^{2}
$$

Since we have to sum over the different vortex configurations $\theta_{\text {vort }}$, the system now appears to describe a gas of classical charged particles interacting via a two-dimensional Coulomb potential and globally neutral [12] (the neutrality constraint comes from the infrared divergence in the $\theta$ two-point function). From one's intuition of a Coulomb gas, one infers that these charges are bound into dipoles by a two-dimensional Coulomb potential and thus form a dielectric medium at low temperature. At high temperature, the charges instead form a plasma which has a finite correlation length due to Debye screening.

The continuum action (5) thus has a line of fixed points, $0>\kappa \geq \kappa^{*}$. To investigate the theory at all values of $\kappa$, one may then choose a lattice action, which has to preserve the essential periodicity property of the continuum action and have a local minimum at $\theta=0$. The most general form of the partition function is 15.

$$
Z=\int \prod_{x} \frac{d \theta(x)}{2 \pi} \prod_{(x y)} \sum_{m(x, y)=-\infty}^{\infty} e^{-J(m(x, y))+i m(x, y)(\theta(x)-\theta(y))}
$$

where the sum is over near neighbours $(x y)$ The function $J$ must be even and have the asymptotic behaviour $J(m) \propto m^{2}$, so that the Gaussian action (5) is recovered in the naive continuum limit.

The simplest choice is the so-called XY model [8]:

$$
S_{\mathrm{xy}}=K_{\mathrm{xy}} \sum_{x, \mu}\left[1-\cos \left(\theta(x)-\theta\left(x+a \hat{e}_{\mu}\right)\right)\right] .
$$

This corresponds to $J(m)=-\log I_{m}\left(K_{\mathrm{xy}}\right)$, where $I_{m}$ is the modified Bessel function. At very small $K_{\mathrm{xy}}$ it is easy to see by strong coupling expansion 12] that the mass gap is given by $\log 1 / K_{\mathrm{xy}}$. When the transition is approached from the high-temperature phase, the correlation length in lattice units diverges as

$$
\xi=\xi_{o} \exp \left(b \sqrt{\frac{K_{\mathrm{xy}}^{*}}{K_{\mathrm{xy}}^{*}-K_{\mathrm{xy}}}}\right),
$$

where the functional form is universal, while $\xi_{o}, b$ and $K_{\mathrm{xy}}^{*}$ are not. At very large $K_{\mathrm{xy}}$, the naive continuum limit (5) is the correct one with $\kappa=K_{\mathrm{xy}}$.

Another standard partition function, with $J(m)=\frac{m^{2}}{2 K_{\mathrm{v}}}$, is the Villain model [11]:

$$
\begin{aligned}
Z_{\mathrm{v}} & =\int \prod_{x} \frac{d \theta(x)}{2 \pi} \prod_{x, \mu} z\left(\theta(x)-\theta\left(x+a \hat{e}_{\mu}\right)\right), \\
z(\theta) & =\sqrt{2 \pi K_{\mathrm{v}}} \sum_{n=-\infty}^{\infty} \exp \left\{-\frac{K_{\mathrm{v}}}{2}(\theta-2 \pi n)^{2}\right\} .
\end{aligned}
$$


Since the asymptotic behaviour of $J(m)$ in the XY-model is $m^{2} / 2 K_{\mathrm{xy}}$, at large $K_{\mathrm{v}}$ the Villain probability distribution becomes identical to the XY probability distribution with $K_{\mathrm{v}}=K_{\mathrm{xy}}$. Thus the continuum limit is again (5) with $\kappa=K_{\mathrm{v}}$ in that regime. In general, the vortices decrease the effective $\kappa$ (defined as the $\kappa$ that would give the same two-point correlation function in the Gaussian approximation [14]). If $R_{o}<a$ is the radius of the vortex-core, the relation is

$$
\frac{1}{\kappa_{\mathrm{eff}}}=\frac{1}{K_{\mathrm{v}}}+\frac{\pi^{2}}{2} \frac{1}{K_{\mathrm{v}}-2 / \pi}\left(\frac{R_{o}}{a}\right)^{2 \pi K_{\mathrm{v}}} .
$$

Thus the correction is exponentially small at large $K_{\mathrm{v}}$.

A duality transformation brings the Villain partition function into the following form, defined on a dual lattice:

$$
Z_{v}=\int \prod_{x} d \phi(x) \sum_{m(x)=-\infty}^{\infty} \exp \left\{-\frac{1}{2 K_{\mathrm{v}}} \sum_{x, \mu}\left(\phi(x)-\phi\left(x+a \hat{e}_{\mu}\right)\right)^{2}+2 \pi i \sum_{r} m(x) \phi(x)\right\},
$$

where $m(x)$ is a dynamical variable taking integer values. It can be shown [26] to be equivalent to the (naively discretised) sine-Gordon model

$$
S_{\mathrm{SG}}=\sum_{x}\left[\frac{1}{2} \sum_{\mu}\left(\phi(x)-\phi\left(x+a \hat{e}_{\mu}\right)\right)^{2}-\frac{m_{o}^{2}}{\beta^{2}} \cos \{\beta \phi(x)\}\right] .
$$

When the fugacity of vortices is small (this is universally the case on an RG trajectory close to the continuum for $\left.\kappa>\kappa^{*}\right)$, one need keep only the terms $m(x)=0, \pm 1$ in (15) and the bare lattice parameters are then related by [12]

$$
\beta=2 \pi \sqrt{K_{\mathrm{v}}}, \quad m_{o}^{2}=8 \pi^{2} K_{\mathrm{v}} e^{-\pi^{2} K_{\mathrm{v}} / 2} .
$$

What is relevant to our discussion is that the continuum limit of the sine-Gordon model at

$$
\beta^{*}=\sqrt{8 \pi}
$$

can be used to describe the Kosterlitz-Thouless transition [17, 22].

The sine-Gordon model is exactly solvable in the continuum [16] by bootstrap methods for $0 \leq \beta^{2}<8 \pi$, in which range the potential term is relevant [13]; $m_{o}$ renormalises but the coupling $\beta$ does not 13 . Remarkably, there is even an exactly solvable (lightcone lattice) regularisation of the model [5].

\section{$3 \quad$ The effective theory for the QCD string}

We briefly remind the reader of the effective theory for the QCD string [6]. It is in principle capable of predicting the splittings (and the degeneracies) between low-lying energy levels of the flux-tube, once a few low-energy constants have been determined. So it is a low-energy effective theory with a finite UV-cutoff set by the string tension. Indeed, at energies of that order, once expects the internal degrees of freedom of the string to become excited.

This theory is specified by a partition function associated with an action living on the worldsheet $R \times L$ of the string. The connection with the gauge theory observables is 
that the Polyakov loop correlation function $\left\langle P_{t}(0)^{*} P_{t}(x)\right\rangle$ with $|\vec{x}|=R$ is proportional to this partition function. The latter may be considered as the partition function of a non-renormalisable Euclidean quantum field theory in $(1+1)$ dimensions, on a spatial 'volume' of linear size $R$ and at temperature $T=1 / L$; or, alternatively, as a statistical mechanics system living on a two-space-dimensional lattice with a lattice spacing of order $\sigma^{-1 / 2}$ (which has nothing to do with the lattice on which the gauge theory may or may not be regulated).

The field living on the worldsheet $\left(0 \leq z_{0} \leq L, 0 \leq z_{1} \leq R\right)$ is a bosonic field $h$ with $D-2$ components. Since it represents the displacement of the world sheet from the classical configuration, it has engineering dimension of length. The Polyakov loops are the propagators of static quarks, so we are discussing the open-string case, and this is reflected in the effective theory by imposing Dirichlet boundary conditions on $h$ at $z_{1}=0$ and $z_{1}=R$. The action of the effective theory is

$$
S=\sigma R L+\mu L+S_{0}+S_{1}+S_{2}^{(1)}+S_{2}^{(2)}+\ldots
$$

where the Gaussian action

$$
S_{0}=\frac{\tilde{\sigma}}{2} \int d^{2} z \partial_{\mu} h \cdot \partial_{\mu} h
$$

gives the leading contribution to the string spectrum at large $R$. Each higher-order term is multiplied by an unknown dimensionless constant $\tilde{c}_{k}^{(i)}$, times $\tilde{\sigma}$; some of them vanish/are constrained by the open-closed string duality []. The lower index on the action terms above indicate how many more derivatives of $h$ they contain than $S_{0}$. The parameter $\tilde{\sigma}$ controls the amplitude of the fluctuations around the classical solution: in this form, the whole action of the effective theory is multiplied by it. Of course, $\tilde{\sigma}$ is of the same order as $\sigma$ itself, and the fact that we are to treat the latter as large (it is the UV cutoff) shows that we are in fact doing a semi-classical calculation. But if we introduce the canonically normalised field $\phi=\sqrt{\tilde{\sigma}} h, \tilde{\sigma}$ is absorbed into the lowenergy constants, $c_{k}^{(i)} \equiv \tilde{c}_{k}^{(i)} /(\tilde{\sigma})^{k / 2}$. The value of $\tilde{\sigma} / \sigma$ is not observable here: if for instance the low-lying energy levels are determined from lattice simulations, one will simply determine the dimension-full constants $c_{k}^{(i)}$ in units of $\sigma$.

It should be noted that for any relativistic bosonic string, one has in fact $\tilde{\sigma}=\sigma$ : the amplitude of Gaussian fluctuations are dictated by the same string tension that determines the energy of the classical configuration. It appears however that one cannot test this property for the flux-tube by measuring the low-energy spectrum in infinite transverse dimensions. From now on we drop the tilde on $\sigma$, but it is good to keep the distinction in mind.

\section{The effect of a periodic transverse dimension}

We now consider the effective theory describing the Polyakov loop correlator, for simplicity, in $D=3$ dimensions - we will later return to the $D=4$ situation. The transverse dimension (the one in which the unique component of $h$ can fluctuate) is periodic, with period $L_{1} \equiv L_{\perp}$ :

$$
\left\langle P_{3}(0) P_{3}^{*}\left(R \hat{e}_{2}\right)\right\rangle \equiv e^{-V\left(R, L_{\perp}\right) L}, \quad L_{3} \equiv L \gg R
$$


How is one to generalise the effective string theory discussed in the previous section to include the effect of the periodicity of the transverse dimension?

The general requirements on the effective action (described in detail in [4]) that lead to the form (20) must be kept, but $h$ is now a periodic variable: $h+L_{\perp}$ must be identified with $h$. It is then convenient to rescale $h$ according to

$$
h=\frac{L_{\perp} \theta}{2 \pi} .
$$

Because of this periodicity, a convenient way to write the most general partition function is to formulate it on a lattice with lattice spacing of order $1 / \sqrt{\sigma\left(L_{\perp}\right)}$. Working on a lattice is equivalent to working with a continuum effective action with a fixed momentum cutoff [23]. Our Ansatz for the effective partition function is

$$
\begin{aligned}
Z\left(L_{\perp}, L, R\right) & =e^{\sigma\left(L_{\perp}\right) R T+\mu T} \int \prod_{x} \frac{d \theta(x)}{2 \pi} \prod_{(x y)} \sum_{m(x, y) \in Z} e^{-J\left(L_{\perp}, m(x, y)\right)+i m(x, y)(\theta(x)-\theta(y))} \\
J\left(L_{\perp}, m\right) & \sim m^{2} /(2 K), \quad m \rightarrow \infty .
\end{aligned}
$$

where $(x y)$ is a pair of nearest neighbours and

$$
K \equiv \frac{\sigma\left(L_{\perp}\right) L_{\perp}^{2}}{(2 \pi)^{2}}
$$

The naive continuum limit is then given by

$$
S_{o}=\frac{K}{2} \int d^{2} z \partial_{\mu} \theta \partial_{\mu} \theta .
$$

Interactions over a few lattice spacings are possible but should not affect the universal properties of the system, as long as the naive continuum limit is the one given in (25). The simple application of the Kosterlitz-Thouless analysis leads to the following scenario as a function of $L_{\perp}$ (assuming $R, L$ very large):

- for large $L_{\perp}$ (low-temperature phase of the XY model), the Gaussian approximation is good, and the periodicity of $\theta$ plays no role for the long-distance properties of the theory. In particular, the mass gap vanishes and the universal part of the Casimir energy of the system remains exactly $\frac{-\pi}{24 R}$.

- as $L_{\perp}$ decreases, the vortices can affect the non-universal contributions to the energy levels.

- when $L_{\perp}$ reaches $L_{\perp}^{*}$, vortices on the worldsheet become relevant and the KosterlitzThouless transition takes place. The KT estimate of $L_{\perp}^{*}$ is given by

$$
L_{\perp}^{*} \simeq \sqrt{\frac{8 \pi}{\sigma\left(L_{\perp}\right)}} .
$$

- for $L_{\perp}<L_{\perp}^{*}$, the theory has a finite mass gap $m$. The Casimir energy if of order $e^{-2 m L}$, and the fluctuation along this dimension is effectively 'switched off'.

- When $L_{\perp} \ll L_{\perp}^{*}$, the strong coupling expansion (see e.g. [12]) tells us that to leading order in $\kappa, m=-\log \kappa$. 
It can be shown [14 that the KT transition takes place at somewhat larger $L_{\perp}$ than the KT estimate (26), because of the disordering effect of vortices. $K^{*}$ can be extracted from lattice simulations: first the function $\sigma\left(L_{\perp}\right)$ must be computed, and then $K^{*}$ can be obtained from the determination of $L_{\perp}^{*}$. It should be noted that it is a non-universal quantity; for the square-lattice XY-model, it was found in numerical simulations [18] that $\kappa^{*}=1.1199(1)$, relatively far from the Kosterlitz-Thouless estimate of $2 / \pi \simeq 0.6366$.

Physical interpretation of vortex configurations It is useful to complement the machinery of two-dimensional statistical mechanics with some more intuitive considerations. The action (23) is a saddle-point expansion around the classical string solution which connects a quark to an antiquark separated by distance $R$. Clearly, there are also classical solutions with a net winding number around the compact transverse dimension. They have energy

$$
E_{\mathrm{cl}}(n)=\sigma\left(L_{\perp}\right) \sqrt{R^{2}+n^{2} L_{\perp}^{2}} \simeq E(n=0)+\frac{\sigma\left(L_{\perp}\right) L_{\perp}^{2} n^{2}}{2 R}, \quad R \gg L_{\perp} .
$$

The $n=1$ classical solution becomes as light as the first Gaussian excitation of the $n=0$ solution when $\sigma\left(L_{\perp}\right) L_{\perp}^{2}=2 \pi$. The different classical configurations can only be connected if the string goes through more energetic configurations. Therefore such transitions are classically forbidden tunnelling processes. Quantum mechanically, one expects the tunnelling to become a frequent fluctuation when the zero-point energy of the $n=0$ vacuum is of same order as the gap $E(1)-E(0)$. In this context, the worldsheet direction of size $R$ is interpreted as the space-direction, and that of size $L$ as the Euclidean time direction in a path integral treatment of the quantum-mechanical string. A vortex being a point-like object on the worldsheet, it corresponds to a process of the string describing a transition from a state at $t=-\infty$ to another state at $t=+\infty$. As long as we are considering the asymptotic limit $L \rightarrow \infty$, only vortex-antivortex configurations have a finite free energy in the 2d QFT. By writing the solution (6) with a single $v \bar{v}$ pair explicitly, it is easy to see that a worldsheet containing a $v \bar{v}$ pair describes the 'life' of a string that had winding number $n=0$ at $t=-\infty$, goes through a state with winding number \pm 1 , and returns to $n=0$ classical state (see Fig. (1).

One may find the discussion analogous to the discussion of instantons in the $4 \mathrm{D}$ infinite volume gauge theory. The (anti)vortices correspond to (anti)instantons, the classical configurations of the string correspond to the configurations of the gauge field at fixed $t$ where the mapping from the sphere $S^{2}$ at spatial infinity to $\mathrm{SU}(2)$ has a definite winding number. A difference is that, due to infrared divergences, in the $2 \mathrm{~d}$ system only configurations satisfying $\sum_{m} m\left(n_{m}-\bar{n}_{m}\right)=0$ are allowed, where $n_{m}\left(\bar{n}_{m}\right)$ is the number of (anti)vortices of strength $m$ in the configuration. By contrast, there are configurations of the 4D gauge theory with a net topological charge which have a finite Euclidean action.

Effect of the vortices on the string spectrum at $L_{\perp}>L_{\perp}^{*} \quad$ For $L_{\perp}>L_{\perp}^{*}$, we are in the low-temperature phase of the XY model, and vortices correspond to an irrelevant operator. Therefore they do not affect the universal $1 / R$ correction, which is determined by the canonical kinetic term, the only renormalisable one compatible of the symmetry requirements on the effective action. 

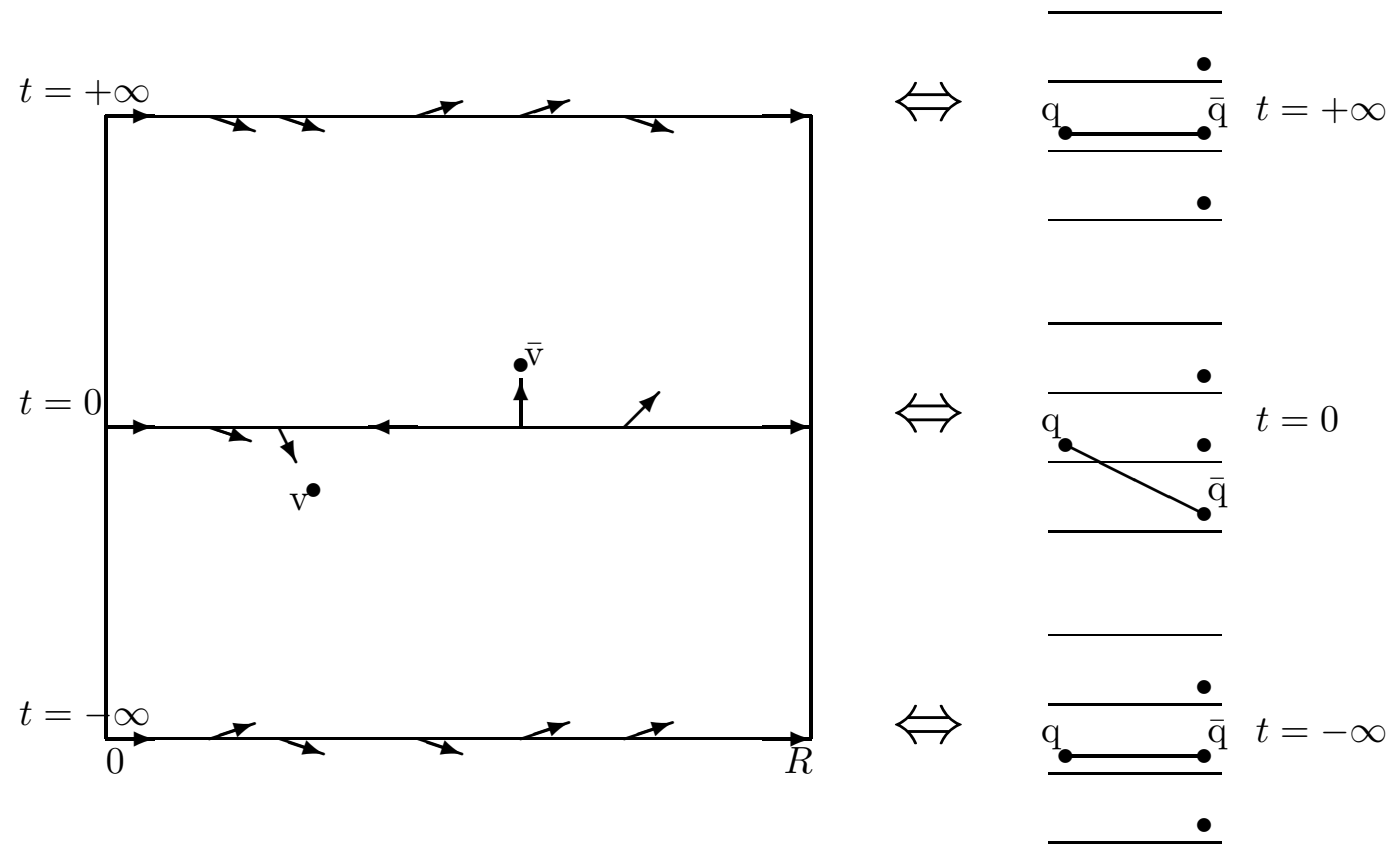

Figure 1: Physical interpretation of the vortices: the left-hand side, where $\theta$ is represented as a unit vector $(\cos \theta, \sin \theta)$, illustrates that the appearance of a vortex at time $t$ 'followed' by an antivortex at time $\bar{t}$ induces one unit of clockwise winding of the variable $\theta\left(t_{s}, x\right)$ as $x$ varies from 0 to $R$ on a timeslice at time $t_{s}$ located between $t$ and $\bar{t}$. Right, the corresponding interpretation for the string on the covering space of the torus: the string has non-trivial winding number along the transverse dimension in the interval $[t, \bar{t}]$ and trivial winding number elsewhere. 
More generally, $E_{C}(R) R$ and $E_{1}(R) R$, the Casimir energy and the energy gap to the first string excitation, are low-energy physical observables in the fixed-cutoff worldsheet quantum field theory; in fact, the latter quantity was used as a definition of the renormalised coupling in the XY-model in [21]. According to Symanzik's theory on the approach to the continuum limit of physical observables, irrelevant operators give a contribution which is suppressed by the lattice spacing to the power of their scaling dimension minus the number of space-time dimensions [23. In particular the higherderivative interactions on the worldsheet give contributions to the energy levels which are suppressed with respect to the $1 / R$ term by $1 / \sqrt{\sigma\left(L_{\perp}\right)}$ to the power of the number of derivatives of the irrelevant operator, minus two. Now as long as the theory is scale invariant, dimensional analysis tells us that the power of $1 / R$ that suppresses the contribution of an irrelevant operator to $E(R) \cdot R$ must be this same power. For instance, $\left(\partial_{\mu} \phi\right)^{2}\left(\partial_{\nu} \phi\right)^{2}$ contributes as $1 /\left(\sigma\left(L_{\perp}\right) R^{2}\right)$ to $E \cdot R$, if $E$ is a string energy level. Thus the parametric form of the energy levels computed in [6] could be guessed on general grounds, while relating the coefficients to the action and computing the string degeneracies requires an explicit calculation. Similarly the vortex-operator gives a suppressed contribution. Its scaling dimension, as computed in the Gaussian theory $S=\frac{\kappa}{2} \int d^{2} x\left(\partial_{\mu} \theta\right)^{2}$, is [15]

$$
x_{\mathrm{v}}=\pi \kappa .
$$

Now, since the free energy of a single vortex is divergent in the infrared, the singlevortex contribution to the partition function vanishes in the thermodynamic limit of the $2 \mathrm{~d}$ system [15]. The first contribution comes from a vortex - anti-vortex pair, so that the vortex contribution to the string ground state energy is suppressed by $1 / R$ to the power $2 x_{\mathrm{v}}-2$ :

$$
\Delta E\left(L_{\perp}, R\right)=\frac{C}{R}\left(\sigma\left(L_{\perp}\right) R^{2}\right)^{1-\pi \kappa}
$$

The peculiarity is that the power varies continuously with the transverse size. We note that it can also be obtained by using the connection with the sine-Gordon model and the fact that the scaling dimension of the $\cos \beta \phi(x)$ operator is $\frac{\beta^{2}}{4 \pi}[13$.

We now discuss the relation between $\kappa$ and $L_{\perp}$. The lattice theory, at lattice spacing $a \equiv 1 / \sqrt{\sigma\left(L_{\perp}\right)}$, is initially parametrised by the coupling $K \equiv \sigma\left(L_{\perp}\right) L_{\perp}^{2} / 4 \pi^{2}$. Under the renormalisation group the coupling $K$ evolves as a function of $\mu a$, where $\mu$ is a renormalisation scale, in order to keep the long-distance physics constant. Under coarsegraining, the theory flows into a Gaussian fixed point, with a Gaussian coupling $\kappa \equiv$ $\lim _{\mu a \rightarrow \infty} K(\mu a)$, which is a non-universal function of the initial $K$. All we can expect is that $\kappa$ is monotonously increasing in $K$.

To determine the RG flow of $K$ in a given model, we have to choose a physical quantity that we keep fixed as the lattice spacing is varied. One possibility is to choose the "spin-spin" correlation function, $\left\langle e^{i(\theta(0)-\theta(x))}\right\rangle$, which in the Gaussian theory behaves as $C(a /|x|)^{1 / 2 \pi \kappa}$ at large distances. In the lattice theory at lattice spacing $a$, the long distance behaviour of this correlator will also decay algebraically, with a certain exponent $b$. Then the renormalised $K(\mu a)$ can be set to $1 / 2 \pi b$. In the Villain (also called 'periodic Gaussian') model, this computation can be carried out explicitly (see section 2, Eq. 14). At large $K=K_{\mathrm{v}}$, the shift from $K$ to $K(\mu a)$ is exponentially small in $K$. In that specific model, the vortices are the only origin of renormalisation. The exponential suppression of their effect is a universal fact, because their fugacity is of order $y=e^{-c K}$, where $c$ is 
however a non-universal quantity representing the core energy of a vortex. In general, there is in addition the more usual renormalisation of $K$ due to operators containing a higher number of derivatives. These effects are of order one. So although the scaling dimension of the vortex operator is a non-universal quantity, at large $K$, we have

$$
\kappa=K+O(1)
$$

Properties of the worldsheet phase transition We now proceed to discuss the universal signatures of the KT transition, from the point of view of string observables.

A first point to note is that the critical value of $\kappa^{*}=2 / \pi$ is universal (while that of $K^{*}$ is not). Therefore the vortex-antivortex pair contribution to the string energy levels reaches

$$
\Delta E \propto 1 / \sigma\left(L_{\perp}^{*}\right) R^{3}
$$

at the KT transition. At the KT point, the fluctuation-field correlations on the worldsheet read, for $\|x\| \gg 1 / \sqrt{\sigma\left(L_{\perp}\right)}$,

$$
\langle h(0) h(x)\rangle=-\left(\frac{L_{\perp}}{4 \pi}\right)^{2} \log \|x\| .
$$

However the vortex operator becomes relevant at that point, and (anti)vortices start to proliferate.

If one is approaching $L_{\perp}^{*}$ from below, that is, from the 'high temperature' phase of the XY model, another universal property of the KT phase transition is the rapidly vanishing mass gap

$$
m \sim m_{0} \exp \left(-b \sqrt{\frac{K^{*}}{K^{*}-K}}\right) .
$$

$m_{0}, K^{*}$ and $b$ are non-universal quantities; but given the function $\sigma\left(L_{\perp}\right)$, this translates through Eq. 24 into a robust prediction for the functional form of $m$ as a function of $L_{\perp}$ close to the phase transition. $\left(\sigma\left(L_{\perp}\right)\right.$ is defined by the asymptotic static force; we are assuming here that $\sigma\left(L_{\perp}\right)$ also governs the quantum fluctuations (see section 3).) So the KT nature of the transition can in principle be tested, but we note that in practice the range where the scaling (33) holds is typically very small [15].

When a mass gap $m$ appears, the Casimir energy must decrease exponentially in $m L$ when the product is large. For a theory of free, neutral massive bosons with Dirichlet boundary conditions, one can indeed show (see Appendix A) that

$$
E_{C}(L)=-\frac{1}{4} \sqrt{\frac{m}{\pi L}} e^{-2 m L}+O\left(e^{-4 m L}\right)
$$

For the current theory, which contains vortices and anti-vortices, one expects twice this expression; and to obtain the corresponding expression for periodic boundary conditions, one can apply the rule [20]

$$
E_{C}(L)_{\text {periodic }}=2 E_{C}\left(\frac{L}{2}\right)_{\text {Dirichlet }} .
$$

The Casimir energy is thus smaller than any power of $1 / L$, and the effective central charge vanishes. So the phase transition of the effective theory driven by vortices explains 
the disappearance of the Lüscher correction as a transverse dimension is reduced in size. Although the vortices are not free, in the sine-Gordon model they correspond to solitons, which are the only one-particle states for $\beta^{2}>4 \pi$, where $\beta^{2} \simeq \sigma\left(L_{\perp}\right) L_{\perp}^{2}$. Their S-matrix is known exactly [16], and the Casimir energy can be obtained from an exact non-linear integral equation [5]: in a periodic box, it reads

$$
E_{C}(L)=-\frac{2 m}{\pi} K_{1}(m L)+\sqrt{\frac{\pi}{m L}} e^{-2 m L}\left[\frac{1+\sqrt{2}}{2}+O\left(\frac{1}{\sqrt{L}}\right)\right] .
$$

The leading term is identical with the free, complex, scalar field with the same periodic boundary conditions. Thus this analytic form is universal, for a given mass gap $m$.

The spread of the cross-over for a finite-length string Strictly speaking, the worldsheet statistical mechanics system cannot have a phase transition for a finite-length string, instead the latter is generically smoothed out into a cross-over. Decreasing $L_{\perp}$ a little below $L_{\perp}^{*}$ leads to a suppression of the $1 / R$ term, which becomes exponential in $m_{0} R$ within

$$
\frac{\delta K}{K^{*}}=\frac{b^{2}}{\log ^{2} m_{0} R} .
$$

From then on, this transverse dimension is effectively "switched off" from the string dynamics. The argument above shows that the width in $K$ (and presumably also in $L_{\perp}$ ) of the smoothed-out phase transition is only logarithmically suppressed in the string length, a distinctive signature of the KT nature of the transition.

Since $m_{0}$ is expected to be of order $\sqrt{\sigma\left(L_{\perp}\right)}$, what we just learnt has the practical consequence that $R$ must be exponentially large in the correlation length of the underlying gauge theory, for this cross-over to be observed at all. This might well explain why these effects were not observed in the numerical studies [2, 1] of spatial torelon masses in $2+1$ dimensional gauge theories.

The transition region for a finite-length string No matter how long the string is, for $L_{\perp}$ sufficiently close to $L_{\perp}^{*}$ the mass gap becomes much smaller than $1 / R$. To get an idea of how the Casimir energy of the string then looks like, we compute in the appendix the leading corrections in the small $m R$ regime to the conformal field theory value $-\pi / 24 R$ in the neutral free scalar field case:

$$
E_{C}=-\frac{\pi}{24 R}+O(m)+O\left(m^{2} R \log (m R)\right) . \quad\left(\sigma^{-1 / 2}\left(L_{\perp}\right) \ll R \ll m^{-1}\right)
$$

More precisely, in terms of the static force, we have

$$
\left|F\left(L_{\perp}, R\right)\right|=\sigma\left(L_{\perp}\right)+\frac{\pi}{24 R^{2}}+\frac{m^{2}}{4 \pi} \log m R+\ldots \quad\left(\sigma^{-1 / 2}\left(L_{\perp}\right) \ll R \ll m^{-1}\right)
$$

It is effectively as if the string tension had a logarithmic dependence on $R$ in this regime.

In the case of periodic boundary conditions, the leading term in $E_{C}$ would be $-\frac{\pi}{6 R}$. This is also the outcome of an exact calculation in the sine-Gordon theory [5]. Interestingly, in this regime the solitons and anti-solitons do not lead to a doubling of the effective central charge; on short distance scales, fluctuations are of the "spin wave type" dictated by the kinetic term of a real scalar field. The Casimir energy of the sine-Gordon model thus interpolates between the Casimir energy of a free complex scalar field in the infrared and a free real scalar field in the ultraviolet. 


\subsection{Additional transverse dimensions \& applications}

So far we discussed the dynamics of a single transverse dimension, in other words we were dealing with a three-dimensional gauge theory. Now we return to the four-dimensional case, where the two components of the worldsheet fluctuation field $h$ are periodic, the case of relevance to standard numerical simulations.

The simple but key point to note is that the two components are decoupled in the quadratic, renormalisable action (20) responsible for the Lüscher term. Therefore the universal contributions to the string energy levels just add up. Also the existence of topological configurations in one component is unaffected by the presence of the other component. In general, the two XY-type models for the two components are coupled by 'irrelevant' terms. This implies in particular that the energy of a vortex-antivortex 'dipole' of size $L_{v \bar{v}}$ receives contributions of order $1 /\left(\sigma\left(L_{\perp}\right) L_{v \bar{v}}^{2}\right)$. This however does not affect the Kosterlitz-Thouless criterion for the occurrence of a phase transition driven by the vortices. We therefore do not expect the KT nature of the worldsheet phase transition to be affected by an additional fluctuation component. Thus, if starting from the $4 \mathrm{D}$ theory, we successively reduce two dimensions, the latter get in turn 'switched off' (from the point of view of contributing to the central charge) at two different KT phase transitions. If we reduce them simultaneously, there presumably are two nearby transitions; this situation however deserves a more detailed investigation.

A convenient way to locate the worldsheet KT transition in Monte-Carlo simulations of the lattice gauge theory by working at a relatively small $L_{1} \equiv L_{\perp}<L_{\perp}^{*}$ is to measure the static quark potential off-axis, say at an angle $\alpha:\left\langle P_{4}(0) P_{4}^{*}\left(R \cos \alpha \hat{e}_{3}+R \sin \alpha \hat{e}_{1}\right)\right\rangle$. The effective periodicity of the worldsheet fluctuation field is then given by the substitution $L_{\perp} \rightarrow L_{\perp}(\alpha) \equiv L_{\perp} / \cos \alpha$. By measuring the static potential $V_{\alpha}(R)$, one can in principle locate the $\alpha^{*}$ where the Lüscher term drops by a unit of central charge. One can then check in an independent simulation that the transition is also seen when setting $L_{\perp}=L_{\perp}\left(\alpha^{*}\right)$ and measuring the static potential on-axis. This would test that the transition is really driven by the periodicity of the fluctuation field $h(z)$.

\section{$5 \quad$ Summary and outlook}

We have studied an effective string theory for the flux-tube in $\mathrm{SU}(N)$ gauge theories in three and four compact dimensions. Let us summarise the outcome of our investigation of the Polyakov loop correlator when one transverse dimension is periodic and of size $L_{\perp}$. The periodic component of the fluctuation field is described by an action of the $\mathrm{XY}$-model type. It follows that

- there is a phase transition of the worldsheet field theory at $L_{\perp}^{*}=O\left(\sigma^{-1 / 2}\right)$ where the periodic component acquires a mass gap and hence the central charge drops by one unit;

- it is a Kosterlitz-Thouless phase transition:

- approaching $L_{\perp}^{*}$ from below, the mass gap $m\left(L_{\perp}\right)$ goes to zero in a universal way in terms of $K \equiv \sigma\left(L_{\perp}\right) L_{\perp}^{2} / 4 \pi^{2}$ as given by Eq. 33. Once $\sigma\left(L_{\perp}\right)$ is independently determined, we have a prediction for the functional form of $m\left(L_{\perp}\right)$. 
- in the large $L_{\perp}$ phase, there are (strongly suppressed) corrections to the string energy levels associated with the periodicity; they come in powers of $1 / R$ which vary continuously and increase monotonously with $L_{\perp}$; these corrections are exactly of order $1 / R^{3}$ at $L_{\perp}=L_{\perp}^{*}$.

We conclude by mentioning some natural extensions of the investigations carried out in this paper.

1. It is also possible to study the QCD string when one transverse dimension is finite with Dirichlet boundary condition for the gauge field, as in the Schrödinger functional (see for instance [24]). The first question to address is what this boundary condition translates into for the worldsheet degrees of freedom; presumably it introduces a cutoff in the amplitude of the fluctuations. Unlike periodic boundary conditions, this cutoff will affect even the "spin waves" of the worldsheet. It is known from the discussion of the roughening phenomenon in lattice QCD that the bosonic string wave functional is Gaussian in the transverse coordinates and that its width grows with the string length $R$ as 25 .

$$
\delta^{2}(R)=\frac{1}{\pi \sigma} \log \frac{R}{R_{o}} .
$$

One would thus expect that until $R$ is $O\left(e^{\sqrt{\sigma} L_{\perp}}\right)$, the effect on the string spectrum is suppressed by $e^{-L_{\perp}^{2} / \delta^{2}(R)}$. At fixed $L_{\perp}$, this offers a chance to verify the quantummechanical broadening of the string.

2. In string theory the duality transformation $L_{\perp} \leftrightarrow \frac{2 \pi}{\sigma L_{\perp}}$ plays an important role. It is clear that a self-dual point at $L_{\perp} \simeq 0.18 \mathrm{fm}$ does not exist in the $\mathrm{SU}(N)$ theory at finite $N$. From the point of view taken in this paper, it is because the string tension and the effective string action varies with $L_{\perp}$, unlike a fundamental string theory.

3. The fact that the QCD flux-tube, when probed at energies well below the scale set by its tension, behaves like a bosonic string living in the same number of dimensions as the gauge theory, is highly remarkable. One might call this the "weak gauge-string equivalence" [6]. Besides this line of investigation, there is the long-standing speculation that the gauge theory itself has an equivalent formulation in string theory (the "strong gauge-string equivalence" [27]). Since the gauge theory is renormalisable, it is a standalone theory which describes phenomena on all scales. Therefore the equivalent string formulation must also make sense at all energy scales, which means that it must be a fundamental string theory. All known fundamental string theories live in a higher number of dimensions.

It is natural to ask what the relation is between the effective four-dimensional string, whose properties can be established through the weak gauge-string correspondence, and the hypothetical, presumably higher-dimensional string theory which behaves like the gauge theory on all scales. One would naively expect the former to arise as the lowenergy effective string obtained by dimensionally reducing the degrees of freedom of the latter, fundamental string theory. If such a relation exists, the worldsheet phase transition of the effective string described in this paper must also affect the fundamental string dynamics, and hence should be reflected in other physical observables of the gauge theory. The gauge theory of course has the 'deconfining' phase transition at 
$L_{\perp} \doteq 1 / T_{c}=O\left(\sigma^{-1 / 2}\right)$, and it is clearly interesting to see whether $L_{\perp}^{*}=1 / T_{c}$. This can be achieved straightforwardly by lattice simulations. Since dimensional reduction in the gauge theory does not work all the way down to $T_{c}$, there is no a priori reason to expect $L_{\perp}^{*}=1 / T_{c}$ : such a coincidence would provide evidence for a relation of the effective QCD string to a fundamental string.

The low-energy constants determined in the weak gauge-string correspondence also provide valuable information on the 'mother' string theory, and the geometry of the space which leads to its dimensional reduction. Their dependence on the number of colours is of particular interest [7, since the equivalence to a string theory is expected to be simpler at large $N$.

4. We note that the Kosterlitz-Thouless worldsheet transition was discussed in the context of the bosonic string, as a fundamental theory in 26 dimensions, by Sathiapalan 9 . The fact that the $O(2)$ nonlinear $\sigma$-model has no fix points for $\kappa<\kappa^{*}$ implies that it cannot be the worldsheet action of a fundamental string theory. The author concludes that in this regime, the string can no longer be considered to be in a flat background, because the vortex condensate contributes to the stress-energy tensor and hence to the space-time curvature. In the case of the QCD string, we have a fixed cutoff, and we have shown that the net effect is a change of the central charge, as measured through the Casimir energy of the string: one component of the worldsheet field effectively acquires a mass. Thus an attractive scenario for a fundamental string is the following. When probed at low energies $E \ll T^{*}$, the Casimir energy appears to be that of a string in lower dimensions, due to the curvature of the target space-time which gives an effective mass to some components of the worldsheet field. But at higher energies, the curvature is irrelevant and the "hidden" components can be excited: the original conformal invariance is restored in this regime. In this way, it is plausible that there exists a fundamental string theory which at low-energies describes the QCD string.

\section{Acknowledgements}

I thank Janos Balog, Rainer Sommer and Peter Weisz for a critical reading of the manuscript and the ensuing discussions which helped me to clarify several issues.

\section{A The Casimir effect for a massive scalar field}

The Casimir energy for a free massive scalar field in $d$ space dimensions with Dirichlet boundary condition in one dimension of size $R$ reads 20 ]

$$
E_{C}(m, R)=\frac{1}{(2 \sqrt{\pi} R)^{d} \Gamma\left(\frac{d}{2}\right)} \int_{0}^{\infty} d t t^{d-1} \log \left(1-e^{-2 \sqrt{t^{2}+m^{2} R^{2}}}\right)
$$

This is the energy per unit of transverse volume. A term proportional to $R$ and a term proportional to $m$ have already been discarded at this stage 20. By expanding the $\operatorname{logarithm} \log (z)$ around $z=1$ and integrating term by term, one obtains $E_{C}$ as a series of modified Bessel functions:

$$
E_{C}(m, R)=\frac{-2}{R^{d}}\left(\frac{m R}{4 \pi}\right)^{\left(\frac{d+1}{2}\right)} \sum_{n=1}^{\infty} \frac{K_{\frac{d+1}{2}}(2 m R n)}{n^{\left(\frac{d+1}{2}\right)}} .
$$


Given the asymptotic large-argument behaviour of the Bessel functions,

$$
K_{\nu}(z) \sim \sqrt{\frac{\pi}{2 z}} e^{-z}, \quad z \rightarrow \infty, \forall \nu
$$

this expression of $E_{C}$ immediately shows that the latter vanishes exponentially fast in $m R$ once this product is large:

$$
E_{C}(m R \rightarrow \infty, R) \sim-\frac{1}{2 R^{d}}\left(\frac{m R}{4 \pi}\right)^{d / 2} e^{-2 m R}
$$

On the other hand, when

$$
\epsilon \equiv m R \ll 1
$$

one naturally expects to recover the Casimir energy of a massless scalar field [20]

$$
E_{C}(m=0, R)=-\frac{1}{(4 \pi)^{\frac{d+1}{2}}} \frac{1}{R^{d}} \Gamma\left(\frac{d+1}{2}\right) \zeta(d+1) .
$$

This is easily checked to be the case, either by using the small-argument expansion of the Bessel functions,

$$
K_{\nu}(z) \sim \frac{\Gamma(\nu)}{2}\left(\frac{z}{2}\right)^{-\nu},
$$

or by contour integration techniques of (41)) (with $m R=0$ ). The leading corrections to this was given in [20] for $d \geq 3$ :

$$
E_{C}(m, R)=E_{C}(m=0, R)+\frac{1}{(4 \pi)^{\frac{d+1}{2}}} \frac{1}{R^{d}} \Gamma\left(\frac{d-1}{2}\right) \zeta(d-1)(m R)^{2}+\ldots
$$

For $d=2$, the leading correction is easily computed by the change of variables $t^{2}=s^{2}-\epsilon^{2}$ in (41):

$$
E_{C}(m, R)=E_{C}(m=0, R)-\frac{m^{2}}{8 \pi}[\log (2 m R)-1]+O(m R)^{3}, \quad d=2 .
$$

We are interested in the Casimir energy of a string, so that $d=1$ and, using Eq. 45 and $\zeta(2)=\frac{\pi^{2}}{6}$, we have the well-known result [4]

$$
E_{C}(m=0, R)=-\frac{\pi}{24 R} .
$$

We now want to compute the leading corrections in $\epsilon$ to this result, which corresponds to the conformal theory. This is a less straightforward calculation, because one cannot simply differentiate (with respect to $\epsilon$ ) the integrand in the first form (41), or the terms of the series in the second form (42), because in both cases the result would diverge. We start off from (42). The key point to note is that as $\epsilon \rightarrow 0$, the series samples the Bessel function in very small steps, so that in can be approximated by an integral: In general, for a smooth function $g(x)$, one would have

$$
\epsilon \sum_{n \geq 1} g(\epsilon n)=\int_{\epsilon / 2}^{\infty} g(x) d x+O\left(\epsilon^{2}\right)
$$


This is however not directly applicable, because the $K_{1}(x)$ function is singular at the origin. We therefore differentiate $E_{C}$ with respect to $\epsilon$ at fixed $R$ :

$$
\begin{aligned}
-2 \pi R \frac{\partial E_{C}(\epsilon, R)}{\partial \epsilon} & =\sum_{n \geq 1} \frac{K_{1}(2 \epsilon n)}{n}+2 \epsilon K_{1}^{\prime}(2 \epsilon n) \\
& =-2 \epsilon \sum_{n \geq 1} K_{0}(2 \epsilon n)
\end{aligned}
$$

where $K_{1}^{\prime}$ is the derivative of $K_{1}$ with respect to its argument. We have used the identity $K_{1}^{\prime}+K_{1} / z=-K_{0}$. We now use the formula, valid for an analytic function,

$$
2 \epsilon \sum_{n \geq 1} f(2 \epsilon n)=\int_{\epsilon}^{\infty} f(x) d x-2 \epsilon \sum_{n \geq 1} \sum_{k \geq 1} \frac{f^{(2 k)}(2 n \epsilon)}{(2 k+1) !} \epsilon^{2 k} .
$$

Now, with $f=K_{o}$,

$$
\int_{\epsilon}^{\infty} K_{o}(x) d x=\int_{0}^{\infty} K_{o}(x) d x-\int_{0}^{\epsilon} K_{o}(x) d x=\frac{\pi}{2}+\epsilon[\log \epsilon-1-\log 2+\gamma]+O\left(\epsilon^{3} \log \epsilon\right) .
$$

The remaining sums in (52) can be evaluated in leading order in $\epsilon$ by using the leading small-argument behaviour of $K_{o}(\epsilon)=-\log \epsilon+O(1)$ :

$$
\sum_{n \geq 1} K_{o}^{(2 k)}(2 n \epsilon) \epsilon^{2 k}=\frac{(2 k-1) ! \zeta(2 k)}{4^{k}}+O\left(\epsilon^{2} \log \epsilon\right)
$$

Gathering the pieces and performing a few elementary integrals, we get

$$
\begin{aligned}
E_{C}(m, R)= & E_{C}(0, R)+\int_{0}^{\epsilon} \frac{\partial E_{C}\left(\epsilon^{\prime}, R\right)}{\partial \epsilon^{\prime}} d \epsilon^{\prime} \\
= & -\frac{\pi}{24 R}+\frac{m}{4}+\frac{m^{2} R}{4 \pi}\left[\log \left(\frac{m R}{2}\right)+\gamma-\frac{3}{2}-\sum_{k \geq 1} \frac{\zeta(2 k)}{4^{k} k(2 k+1)}\right] \\
& +O\left(m^{4} R^{3} \log m R\right)
\end{aligned}
$$

It is clear from the results above that the introduction of a small mass for the scalar field has a stronger effect in lower space dimensions. This is natural since infrared effects tend to be stronger in lower-dimensional field theories. The term proportional to $m^{2} R \log m R$ is the first term of interest, in the sense that it is the leading observable effect in a system with fixed $m$ and varying $R$. It expresses the fact that the bulk free energy of the system has a logarithmic dependence on its size, in the regime $m R \leq 1$.

The cross-over between the small $m R$ and large $m R$ regimes is smooth. The area $-R \int_{0}^{\infty} d \epsilon E_{C}(\epsilon, R)$ is easily computed and amounts to $\zeta(3) / 16$.

\section{References}

[1] B. Lucini and M. Teper, Phys. Rev. D 64 (2001) 105019 arXiv:hep-lat/0107007.

[2] M. Teper, Phys. Lett. B 311 (1993) 223. 
[3] M. Luscher and P. Weisz, JHEP 0207 (2002) 049 arXiv:hep-lat/0207003.

[4] M. Luscher, K. Symanzik and P. Weisz, Nucl. Phys. B 173 (1980) 365; M. Luscher, Nucl. Phys. B 180 (1981) 317.

[5] C. Destri and H. J. De Vega, Nucl. Phys. B 438 (1995) 413 arXiv:hep-th/9407117.

[6] M. Luscher and P. Weisz, JHEP 0407 (2004) 014 arXiv:hep-th/0406205.

[7] H. Meyer and M. Teper, JHEP 0412, 031 (2004) arXiv:hep-lat/0411039.

[8] J. M. Kosterlitz and D. J. Thouless, J. Phys. CC 6, 1181 (1973).

[9] B. Sathiapalan, Phys. Rev. D 35 (1987) 3277.

[10] K. J. Juge, J. Kuti and C. Morningstar, arXiv:hep-lat/0401032; K. J. Juge, J. Kuti and C. Morningstar, Nucl. Phys. Proc. Suppl. 129 (2004) 686 arXiv:hep-lat/0310039.

[11] J. Villain, J. Physique 36 (1975) 581.

[12] J. B. Kogut, Rev. Mod. Phys. 51 (1979) 659.

[13] J. Zinn-Justin, Quantum field theory and critical phenomena, OUP 2002.

[14] C. Itzykson and J.-M. Drouffe, Statistical Field Theory, Vol. I, CUP 1989.

[15] J. Cardy, Scaling and renormalization in statistical physics, CUP 1989

[16] A. B. Zamolodchikov and A. B. Zamolodchikov, Annals Phys. 120 (1979) 253.

[17] D. J. Amit, Y. Y. Goldschmidt and G. Grinstein, J. Phys. A 13 (1980) 585.

[18] M. Hasenbusch and K. Pinn, J. Phys. A 30, 63 (1997) arXiv:cond-mat/9605019.

[19] R. Sommer, Nucl. Phys. B 306 (1988) 180; G. S. Bali, K. Schilling and C. Schlichter, Phys. Rev. D 51 (1995) 5165 arXiv:hep-lat/9409005; P. Pennanen, A. M. Green and C. Michael, Phys. Rev. D 56 (1997) 3903 arXiv:hep-lat/9705033.

[20] J. Ambjorn and S. Wolfram, Annals Phys. 147, 1 (1983); K. A. Milton, The Casimir effect: Physical manifestations of zero-point energy, World Scientific 2001.

[21] M. Luscher, P. Weisz and U. Wolff, Nucl. Phys. B 359 (1991) 221; J. Balog, F. Knechtli, T. Korzec and U. Wolff, Nucl. Phys. B 675, 555 (2003) arXiv:hep-lat/0309028.

[22] J. Balog, J. Phys. A 34, 5237 (2001) arXiv:hep-lat/0011078.

[23] K. Symanzik, Nucl. Phys. B 226 (1983) 187.

[24] M. Luscher, arXiv:hep-lat/9802029.

[25] M. Luscher, G. Munster and P. Weisz, Nucl. Phys. B 180 (1981) 1.

[26] J. Balog, private communication.

[27] A. M. Polyakov, Int. J. Mod. Phys. A 14 (1999) 645 arXiv:hep-th/9809057. 